\title{
Two-dimensional Massive Fermions and the Federbush Model: Space-like vs. Light-front Picture
}

\section{L'ubomír Martinovič*}

Institute of Physics, Slovak Academy of Sciences, Dúbravská cesta 9, 84511 Bratislava, Slovakia

E-mail: fyziluma@savba.sk

\begin{abstract}
The exactly solvable Federbush model, describing two species of massive fermions with vector current - axial-vector current interaction in two dimensions, is studied in terms of light front (LF) space-time and field variables using Hamiltonian framework. The aim is to compare vacuum states and correlation functions constructed from known operator solutions of the field equations with those of the conventional space-like (SL) form of the theory. As a prerequisite, SL and LF treatment of free massive fermion fields is compared and the necessity to replace usual Wick ordering by the "triple-dot ordering" is briefly mentioned within the nonperturbative bosonization of Lehmann and Stehr. Here the main mathematical subtlety is a correct mathematical definition of the exponential function of the functional bilinear in fermion Fock operators. In order to gain an insight to the vacuum structure at a simpler level, the massless Thirring model in the Klaiber's formulation is re-analyzed and its physical vacuum is derived using the Bogoliubov transformation similar to the transformation proposed by Mattis and Lieb in the case of the Luttinger model.
\end{abstract}

LIGHT CONE 2008 Relativistic Nuclear and Particle Physics

July 7-11 2008

Mulhouse, France

\footnotetext{
*Speaker.
} 


\section{Introduction}

There exist striking differences between the conventional space-like (SL) and light-front (LF) forms of field theories. This is true for the mathematical structure as well as for some physical aspects (number and nature of field variables, structure of the Poincare algebra, status of the vacuum states, etc.). What is the relation between the two formulations of quantum field theory (QFT)?

An indication is provided by the perturbative analysis of the Yukawa model [1] which showed that LF Fock operators are represented as complicated superpositions of the creation and annihilation operators of the SL theory. It would be desirable to verify and extend this picture nonperturbatively. This is a highly non-trivial task due to a very complicated mathematical structure of realistic QFT models. A useful idea could be to study exactly soluble models where operator solutions of the field equations are known and the nonperturbative (NP) correlation functions can be calculated. Thus, the role of the vacuum state and of the operator part in both schemes can be compared.

The sketched program is far from being trivial in view of surprising complexities found in exactly solvable SL models (nonperturbative bosonization [2], Federbush correlation functions [3]).

The LF formulation of these models seems to be much simpler, first of all due to the simplified vacuum state. A possible but highly improbable explanation is that the LF formulation is an inconsistent scheme. Perhaps some mistakes have been done in the SL theory: why the correlation functions have been calculated using the Fock vacuum $|0\rangle[4,3]$ ? This state is not an eigenstate of the full Hamiltonian and hence it cannot be the true ground state! On the other hand, the LF Fock vacuum is often the physical ground state, i.e. the eigenstate of Hamiltonian with minimal energy.

In the present contribution, the search of the relationship (a " bridge") between the LF and SL forms of QFT is started in the area of soluble models where one can get complete NP answers and see in details how each scheme works. One of the main ideas will be to write the SL Hamiltonian in terms of composite boson operators. In this way, the interacting terms become bilinear and could be in principle diagonalized by means of a Bogoliubov transformation. Then the true lowestenergy eigenstate will be a transformed Fock vacuum (an exponential state) and the correlation functions should be calculated as its expectation values. The massless Thirring model and (massive) Federbush model will be analyzed in this way. Since some related mathematical complexities (a correctly defined exponential function of a functional bilinear in fermion Fock operators) were studied within the NP bosonization of the free massive SL fermion field [2], its simplified treatment along with the LF version is discussed, too. The hope is that one can eventually arrive at a reconciliation of the physical contents of the SL and LF forms of two dimensional models.

\section{Free massive fermion fields in $D=1+1$}

The Lagrangian of the free massive fermion field in $\mathrm{D}=1+1$ and the general solution of its Dirac equation $\left(i \gamma^{\mu} \partial_{\mu}-m\right) \psi(x)=0$ with $2 \times 2$ matrices $\gamma^{\mu}=\left(\gamma^{0}=\sigma_{1}, \gamma^{1}=i \sigma_{2}\right), \alpha=\gamma^{0} \gamma^{1}$, is

$$
\mathscr{L}=\frac{i}{2} \bar{\psi} \gamma^{\mu} \overleftrightarrow{\partial_{\mu}} \psi-m \bar{\psi} \psi, \quad \psi(x)=N \int d^{2} p \delta\left(p^{2}-m^{2}\right) a(p) u(p) e^{-i p \cdot x}
$$

After $p^{0}$ integration and reinterpretation of negative-energy solutions, it takes the form

$$
\psi(x)=\int \frac{d p}{\sqrt{2 \pi}}\left[b(p) u(p) e^{-i \hat{p} \cdot x}+d^{\dagger}(p) v(p) e^{i \hat{p} . x}\right], \hat{p} \cdot x=E(p) t-p x, E(p)=\sqrt{p^{2}+m^{2}}
$$


with the Fock anticommutators $\left\{b(p), b^{\dagger}(q)\right\}=\left\{d(p), d^{\dagger}(q)\right\}=\delta(p-q)$. The "spinors" are

$$
u(p)=\frac{1}{\sqrt{2 E(p)}}\left(\begin{array}{c}
\sqrt{p^{-}} \\
\sqrt{p^{+}}
\end{array}\right), v(p)=\frac{1}{\sqrt{2 E(p)}}\left(\begin{array}{c}
-\sqrt{p^{-}} \\
\sqrt{p^{+}}
\end{array}\right), p^{ \pm}=E(p) \pm p .
$$

The currents $j^{\mu}(x)=: \bar{\psi}(x) \gamma^{\mu} \psi(x):=\left(\psi^{\dagger} \psi, \psi^{\dagger} \alpha \psi\right), j_{5}^{\mu}(x)=: \bar{\psi}(x) \gamma^{\mu} \gamma^{5} \psi(x):=\left(j^{1}, j^{0}\right)$,

$$
\begin{gathered}
j^{0(1)}(x)=\frac{1}{2 \pi} \int_{-\infty}^{+\infty} \mathrm{d} p \int_{-\infty}^{+\infty} \mathrm{d} q\left\{f_{1(2)}(p, q)\left[b^{\dagger}(p) b(q) e^{i(\hat{p}-\hat{q}) \cdot x}-d^{\dagger}(q) b(p) e^{-i(\hat{p}-\hat{q}) \cdot x}\right]\right. \\
\left.+f_{2(1)}(p, q)\left[b^{\dagger}(p) d^{\dagger}(q) e^{i(\hat{p}+\hat{q}) \cdot x}-b(q) d(p) e^{-i(\hat{p}+\hat{q}) \cdot x}\right]\right\}
\end{gathered}
$$

contain the kinematical functions $f_{1}, f_{2}$ coming from the nontrivial spinor structure:

$$
f_{1(2)}(p, q)=\frac{1}{2 \sqrt{E(p) E(q)}}\left[\sqrt{p^{+} q^{+}} \pm \sqrt{p^{-} q^{-}}\right]
$$

The free-field commutator at unequal times can be straightforwardly calculated,

$$
\begin{aligned}
& {\left[j^{\mu}(x), j^{v}(y)\right]=-i: \bar{\psi}(x) \gamma^{\mu} S(x-y) \gamma^{v} \psi(y):+i: \bar{\psi}(y) \gamma^{v} S(y-x) \gamma^{\mu} \psi(x):} \\
& \quad+\operatorname{Tr}\left\{S^{(-)}(x-y) \gamma^{v} S^{(+)}(y-x) \gamma^{\mu}-S^{(-)}(y-x) \gamma^{\mu} S^{(+)}(x-y) \gamma^{v}\right\} .
\end{aligned}
$$

The fermionic two-point functions $S(x-y)=S^{(+)}(x-y)+S^{(-)}(x-y)$, with $z=x-y$, are

$$
S^{( \pm)}(z)=\frac{i}{2 \pi}\left(i \gamma^{\mu} \partial_{\mu}^{x}+m\right) \int d^{2} p \delta\left(p^{2}-m^{2}\right) \theta\left( \pm p^{0}\right) e^{ \pm i p \cdot z}=\frac{i}{4 \pi} \int \frac{d p}{E(p)}\left(\begin{array}{cc}
m & p^{-} \\
p^{+} & m
\end{array}\right) e^{ \pm i \hat{p} \cdot z}
$$

At $x^{0}=y^{0}$, the last term in (2.5) is the c-number term, $\left[j^{0}(0, x), j^{1}(0, y)\right]=i / \pi \partial_{x} \delta(x-y)$. Does the LF theory agree with the result (2.5) and the above Schwinger term?

The Lagrangian (2.1) takes in terms of the LF fermionic field components $\psi_{2}$ and $\psi_{1}$ the form

$$
\mathscr{L}_{l f}=i \psi_{2}^{\dagger}{\overleftrightarrow{\partial_{+}}}_{\psi_{2}}+i \psi_{1}^{\dagger} \overleftrightarrow{\partial_{-}} \psi_{1}-m\left(\psi_{2}^{\dagger} \psi_{1}+\psi_{1}^{\dagger} \psi_{2}\right)
$$

We are using the representation where $\gamma^{5}=\operatorname{diag}(1,-1)$. The LF Dirac matrices $\gamma^{ \pm}=\gamma^{0} \pm \gamma^{1}$ satisfy the algebra $\gamma^{+} \gamma^{-}+\gamma^{-} \gamma^{+}=4 \hat{1}$ which allows one to define projectors $\Lambda_{ \pm}=1 / 4 \gamma^{\mp}, \Lambda_{+}+$ $\Lambda_{-}=\hat{1}$ leading to the LF form (2.7) of the Lagrangian. The Dirac equation separates into two equations - the dynamical for $\psi_{2}$ and the non-dynamical for the $\psi_{1}$ component, $2 i \partial_{+} \psi_{2}(x)=$ $m \psi_{1}(x), 2 i \partial_{-} \psi_{1}(x)=m \psi_{2}(x)$. The latter can be inverted to express $\psi_{1}$ in terms of $\psi_{2}$ :

$$
\psi_{1}(x)=\frac{m}{2 i} \partial_{-}^{-1} \psi_{2}(x)=\frac{m}{4 i} \int_{-\infty}^{+\infty} d y^{-} \varepsilon\left(x^{-}-y^{-}\right) \psi_{2}\left(x^{+}, y^{-}\right) .
$$

Evidently, the matrix and "spinor" structure plays only a marginal role in the LF case, simplifying the picture considerably, while the matrix algebra in the SL case is in principle as complicated as in $3+1$ dimensions. The LF description is more adequate because there is no spin in one space dimension and the whole machinery of the spinors and matrices is redundant. The fermionic character of $\psi$ field is maintained (two components, anticommutators instead of commutators). 
The field expansion, with $\hat{p} \cdot x=\frac{1}{2} p^{+} x^{-}+\frac{1}{2} \hat{p}^{-} x^{+}, \hat{p}^{-}=\frac{m^{2}}{p^{+}}$,

$$
\psi=\left(\begin{array}{l}
\psi_{1} \\
\psi_{2}
\end{array}\right)=\int_{0}^{\infty} \frac{\mathrm{d} p^{+}}{2 \sqrt{2 \pi}}\left[\left(\begin{array}{c}
\frac{m}{p^{+}} \\
1
\end{array}\right) b\left(p^{+}\right) e^{-i \hat{p} \cdot x}+\left(\begin{array}{c}
-\frac{m}{p^{+}} \\
1
\end{array}\right) d^{\dagger}\left(p^{+}\right) e^{i \hat{p} . x}\right]
$$

leads to the currents

$$
\begin{aligned}
& j^{+(-)}(x)=2: \psi_{2(1)}^{\dagger}(x) \psi_{2(1)}(x):=2 \int_{0}^{\infty} \frac{\mathrm{d} p^{+}}{2 \sqrt{2 \pi}} \int_{0}^{\infty} \frac{\mathrm{d} q^{+}}{2 \sqrt{2 \pi}}\left(\frac{m^{2}}{p^{+} q^{+}}\right)\left[b^{\dagger}\left(p^{+}\right) b\left(q^{+}\right) e^{i(\hat{p}-\hat{q}) \cdot x}-\right. \\
& \left.-d^{\dagger}\left(q^{+}\right) d\left(p^{+}\right) e^{-i(\hat{p}-\hat{q}) \cdot x} \pm b^{\dagger}\left(p^{+}\right) d^{\dagger}\left(q^{+}\right) e^{i(\hat{p}+\hat{q}) \cdot x} \mp b\left(q^{+}\right) d\left(p^{+}\right) e^{-i(\hat{p}+\hat{q}) \cdot x}\right] .
\end{aligned}
$$

One reproduces the commutation relation (2.5). For example, we get

$$
\left[j^{+}(x), j^{+}(y)\right]=4:\left[\psi_{2}^{\dagger}(x) \psi_{2}(y)-\psi_{2}^{\dagger}(y) \psi(x)\right]:\left[S_{22}(z)+S_{22}^{*}(z)\right]+4\left[S_{22}^{2}(z)-S_{22}^{* 2}(z)\right]
$$

where $S_{22}$ is the two-point function, $S_{22}(x-y)=\left\langle 0\left|\psi(x) \psi^{\dagger}(y)\right| 0\right\rangle=1 / 8 \pi \int_{0}^{\infty} d p^{+} e^{-i \hat{p} .(x-y-i \varepsilon)}$ of the dynamical component. The small imaginary part $\pm i \varepsilon$ is crucial for convergence of the integral and for the correct equal LF time limit of (2.11) - the correct c-number Schwinger term is obtained:

$$
\begin{aligned}
& S_{22}\left(\delta, x^{-}-y^{-}\right)=-\frac{m}{4 \pi} \sqrt{\frac{i \delta}{x^{-}-y^{-}-i \varepsilon}} K_{1}\left(m \sqrt{i \delta\left(x^{-}-y^{-}-i \varepsilon\right)}\right)=-\frac{1}{4 \pi\left(x^{-}-y^{-}-i \varepsilon\right)}, \\
& S_{22}^{2}\left(\delta, x^{-}-y^{-}\right)-S_{22}^{* 2}\left(\delta, x^{-}-y^{-}\right)=\left(\frac{-i}{4 \pi}\right)^{2} \frac{1}{\left(x^{-}-y^{-}-i \varepsilon\right)^{2}}-\left(\frac{i}{4 \pi}\right)^{2} \frac{1}{\left(x^{-}-y^{-}+i \varepsilon\right)^{2}}= \\
& =\left(\frac{i}{4 \pi}\right)^{2} \partial_{-}^{x}\left[\frac{1}{x^{-}-y^{-}-i \varepsilon}-\frac{1}{x^{-}-y^{-}+i \varepsilon}\right]=\frac{i}{8 \pi} \partial_{-}^{x} \delta\left(x^{-}-y^{-}\right) .
\end{aligned}
$$

\section{Non-perturbative bosonization of Lehmann and Stehr}

A bosonization of free massive fermion, more general than the perturbative approach of Coleman, was formulated in [2]. It starts from the "integrated current" $\sigma(x)$ defined by

$$
j^{0}(x)=\frac{1}{\sqrt{\pi}} \partial_{1} \sigma(x), j^{1}(x)=-\frac{1}{\sqrt{\pi}} \partial_{0} \sigma(x) \Rightarrow \sigma(x)=\sqrt{\pi} \int_{-\infty}^{x^{1}} d \xi: \psi^{\dagger} \psi:\left(x^{0}, \xi\right) .
$$

Due to $\partial_{\mu} j_{5}^{\mu}=2 i m \bar{\psi} \gamma^{5} \psi$, it satisfies $\partial_{\mu} \partial^{\mu} \sigma(x)=2 i m \sqrt{\pi}: \bar{\psi} \gamma^{5} \psi:$. The task is to find the rhs of the latter equations in terms of $\sigma(x)$. The main object of the study is $E_{\lambda}(x)=\vdots \exp (2 \sqrt{\pi} i \lambda \sigma(x))$ : regularized by the "triple-dot ordering" [5]. The usual normal ordering is not sufficient for bilinears due to extra divergences. Powers in the expansion are defined in terms of

$$
\vdots \sigma\left(x_{1}\right) \ldots \sigma\left(x_{n}\right) \vdots=\sigma\left(x_{1}\right) \ldots \sigma\left(x_{n}\right)-\sum_{r=1}^{n} \sum_{P}\left\langle\sigma\left(x_{i_{1}}\right) \ldots \sigma\left(x_{i_{r}}\right)\right\rangle_{0} \vdots \sigma\left(x_{j_{1}}\right) \ldots \sigma\left(x_{j_{n-r}}\right) \vdots .
$$

as $\vdots \sigma^{n}(x) \vdots=\lim _{x_{1}, \ldots, x_{n} \rightarrow x} \vdots \sigma\left(x_{1}\right) \ldots \sigma\left(x_{n}\right) \vdots$. The first sum indicates partitions of indices $1, \ldots n$ into disjoint subsets with $i_{1}<i_{2}<\ldots<i_{r}, j_{1}<j_{2}<\ldots j_{n-r}$. The final results are

$$
: \bar{\psi} \psi:=-\frac{m}{\sqrt{\pi}} \vdots \sigma \sin (2 \sqrt{\pi} \sigma) \vdots, \quad: \bar{\psi} \gamma^{5} \psi:=\frac{i m}{\sqrt{\pi}} \vdots \sigma[\cos (2 \sqrt{\pi} \sigma)-1] \vdots .
$$

The analysis shows that the LF version is much simpler due to the simplified spinor structure. 


\section{Klaiber's formulation of the massless Thirring model}

The massless Thirring model is defined by the Lagrangian density and the field equations

$$
\begin{gathered}
\mathscr{L}=\frac{i}{2} \bar{\Psi} \gamma^{\mu} \overleftrightarrow{\partial_{\mu}} \Psi-g J_{\mu} J^{\mu}, \\
i \gamma^{\mu} \partial_{\mu} \Psi(x)=-g J^{\mu}(x) \gamma_{\mu} \Psi(x), J^{\mu}=\bar{\Psi} \gamma^{\mu} \Psi, \partial_{\mu} J^{\mu}(x)=0 .
\end{gathered}
$$

The solution is given in terms of the integrated current $j(x)$ and the free field $\gamma^{\mu} \partial_{\mu} \psi(x)=0$ :

$$
\Psi(x)=e^{i(g / \sqrt{\pi}) j(x)} \psi(x), \quad j_{\mu}(x)=\frac{1}{\sqrt{\pi}} \partial_{\mu} j(x), J^{\mu}(x)=j^{\mu}(x) .
$$

Free fields define the solution of the interacting model. The expansion of the $\psi$ field is

$$
\begin{aligned}
& \psi(x)=\frac{1}{\sqrt{2 \pi}} \int d p^{1}\left\{b\left(p^{1}\right) e^{-i p \cdot x}+d^{\dagger}\left(p^{1}\right) e^{i p \cdot x}\right\} u\left(p^{1}\right), \quad p^{0}=\left|p^{1}\right| \\
& \left\{b\left(p^{1}\right), b^{\dagger}\left(q^{1}\right)\right\}=\left\{d\left(p^{1}\right), d^{\dagger}\left(q^{1}\right)\right\}=\delta\left(p^{1}-q^{1}\right), \quad b\left(k^{1}\right)|0\rangle=d\left(k^{1}\right)|0\rangle=0 .
\end{aligned}
$$

The spinor $u\left(p^{1}\right)$ satisfies $(\gamma \cdot p) u\left(p^{1}\right)=0$, where $u^{\dagger}\left(p^{1}\right)=\left(\theta\left(-p^{1}\right), \theta\left(p^{1}\right)\right)$. Vector current can be represented in terms of composite operators $c(k), c^{\dagger}(k)$, having the property $c(k)|0\rangle=0$, as

$$
\begin{aligned}
j^{\mu}(x) & =-\frac{i}{\sqrt{2} \pi} \int \frac{d k^{1}}{\sqrt{2 k^{0}}} k^{\mu}\left\{c\left(k^{1}\right) e^{-i k \cdot x}-c^{\dagger}\left(k^{1}\right) e^{i k \cdot x}\right\}, \\
c\left(k^{1}\right) & =\frac{i}{\sqrt{k^{0}}} \int d p^{1}\left\{\theta\left(p^{1} k^{1}\right)\right)\left[b^{\dagger}\left(p^{1}\right) b\left(p^{1}+k^{1}\right)-d^{\dagger}\left(p^{1}\right) d\left(p^{1}+k^{1}\right)\right]+ \\
& \left.+\theta\left(p^{1}\left(p^{1}-k^{1}\right)\right) d\left(k^{1}-p^{1}\right) b\left(p^{1}\right)\right\} \Rightarrow\left[c\left(p^{1}\right), c^{\dagger}\left(q^{1}\right)\right]=\delta\left(p^{1}-q^{1}\right) .
\end{aligned}
$$

Large part of the paper is devoted to the infrared regularization and to verification of basic properties (Poincar'e invariance, locality) of the regularized n-point correlation functions. They are based on the normal ordered operator solution $\Psi(x)=e^{(i g / \pi) j^{+}(x)} \psi(x) e^{(i g / \pi) j^{-}(x)}$. Hamiltonian was never mentioned. It has the simplest form in terms of the operators $c(k), c^{\dagger}(k)$ :

$$
H_{\text {int }}=\frac{g}{\pi} \int_{-\infty}^{+\infty} d k|k|\left[c(k) c(-k)+c^{\dagger}(k) c^{\dagger}(-k)\right] .
$$

Obviously $|0\rangle$ is not an eigenstate of $H=H_{0}+H_{\text {int }}$. If some unitary $U(\gamma)$ diagonalizes $H$, then $U(\gamma) H U^{-1}(\gamma)|0\rangle=0$ and $U^{-1}(\gamma)|0\rangle$ will be the physical vacuum state. In fact, a Bogoliubov transformation similar to that used in [7] for the Luttinger model does diagonalize the Hamiltonian. The main idea can be illustrated with two degrees of freedom $c_{1}^{\dagger}, c_{2}^{\dagger}$. Choose new operators $C_{1}^{\dagger}, C_{2}^{\dagger}$

$$
C_{1}^{\dagger}=u c_{1}^{\dagger}-v c_{2}, \quad C_{1}=u^{*} c_{1}-v^{*} c_{2}^{\dagger}, \quad C_{2}^{\dagger}=-v c_{1}^{\dagger}+u c_{2}, \quad C_{2}^{\dagger}=-v^{*} c_{1}+u^{*} c_{2}^{\dagger} .
$$

Requiring that $C_{1}, C_{2}$ satisfy the original commutation relations, we find $u=\cosh \gamma, v=\sinh \gamma$. This transformation can be realized in terms of a unitary operator $U(\gamma)=e^{\gamma\left[c_{1}^{\dagger} c_{2}^{\dagger}-c_{2} c_{1}\right]}$ :

$$
U(\gamma) c_{1}^{\dagger} U^{-1}(\gamma)=c_{1}^{\dagger} \cosh \gamma-c_{2} \sinh \gamma, \quad U(\gamma) c_{2}^{\dagger} U^{-1}(\gamma)=-c_{1}^{\dagger} \sinh \gamma+c_{2} \cosh \gamma
$$

Generalization to infinite number of degrees of freedom is simple: work with $\gamma(p)$ and require that the non-diagonal terms in transformed $H_{i n t}$ vanish. Knowing $U(\gamma(p))$, the true vacuum is

$$
|v a c\rangle=U^{-1}(\gamma(p))|0\rangle
$$

One has to calculate the correlation functions using this ground state. 


\section{Federbush model}

The Lagrangian of the Federbush model [6]

$$
\mathscr{L}=\frac{i}{2} \bar{\Psi} \gamma^{\mu} \overleftrightarrow{\partial_{\mu}} \Psi-m \bar{\Psi} \Psi+\frac{i}{2} \bar{\Phi} \gamma^{\mu} \overleftrightarrow{\partial_{\mu}} \Phi-M \bar{\Phi} \Phi-g \varepsilon_{\mu v} j^{\mu} J^{v}, \quad \varepsilon^{\mu v}=-\varepsilon^{v \mu}, \quad \varepsilon^{01}=1
$$

describes two species of the fermion field interacting via specific current-current coupling, where $j^{\mu}=\bar{\Psi} \gamma^{\mu} \Psi, J^{\mu}=\bar{\Phi} \gamma^{\mu} \Phi$. Unlike the closely related massive Thirring model, Federbush model is exactly soluble at the level of operator solutions of the field equations. Using the previous twocomponent spinors in the chiral representation, the coupled field equations

$$
\begin{aligned}
& 2 i \partial_{+} \Psi_{2}(x)=m \Psi_{1}-g J^{-} \Psi_{2}, \quad 2 i \partial_{-} \Psi_{1}=m \Psi_{2}+g J^{+} \Psi_{1}, \\
& 2 i \partial_{+} \Phi_{2}(x)=M \Phi_{1}+g j^{-} \Phi_{2}, \quad 2 i \partial_{-} \Phi_{1}=M \Phi_{2}-g j^{+} \Phi_{1} .
\end{aligned}
$$

are solved in terms of the corresponding free fields and the integrated currents:

$$
\begin{gathered}
\Psi(x)=e^{\frac{i g}{\sqrt{\pi}} \Sigma(x)} \psi(x), \quad \Phi(x)=e^{-\frac{i g}{\sqrt{\pi}} \sigma(x)} \varphi(x), \\
\sigma(x)=\frac{\sqrt{\pi}}{4} \int_{-\infty}^{+\infty} \mathrm{d} z^{-} \varepsilon\left(x^{-}-z^{-}\right) j^{+}\left(x^{+}, z^{-}\right), \quad \Sigma(x)=\frac{\sqrt{\pi}}{4} \int_{-\infty}^{+\infty} \mathrm{d} z^{-} \varepsilon\left(x^{-}-z^{-}\right) J^{+}\left(x^{+}, z^{-}\right) .
\end{gathered}
$$

The field expansions and the quantization conditions at $x^{+}=0$ read:

$$
\begin{aligned}
& \psi_{2}(x)=\int_{0}^{\infty} \frac{\mathrm{d} p^{+}}{2 \sqrt{2 \pi}}\left[b\left(p^{+}\right) e^{-i \hat{p} . x}+d^{\dagger}\left(p^{+}\right) e^{i \hat{p} . x}\right], \quad \varphi_{2}(x)=\int_{0}^{\infty} \frac{\mathrm{d} p^{+}}{2 \sqrt{2 \pi}}\left[a\left(p^{+}\right) e^{-i \hat{p} \cdot x}+c^{\dagger}\left(p^{+}\right) e^{i \hat{p} \cdot x}\right], \\
& \left\{\psi_{2}\left(0, x^{-}\right), \psi_{2}^{\dagger}\left(0, y^{-}\right)\right\}=\left\{\varphi_{2}\left(0, x^{-}\right), \varphi_{2}^{\dagger}\left(0, y^{-}\right)\right\}=\frac{1}{2} \delta\left(x^{-}-y^{-}\right)
\end{aligned}
$$

In Fock representation, the operators (5.4) are expressed in terms of the currents (2.10) as

$$
\begin{aligned}
\sigma(x)=i 2 & \sqrt{\pi} \int_{0}^{\infty} \frac{\mathrm{d} p^{+}}{2 \sqrt{2 \pi}} \int_{0}^{\infty} \frac{\mathrm{d} q^{+}}{2 \sqrt{2 \pi}}\left[\frac{b^{\dagger}\left(p^{+}\right) b\left(q^{+}\right)}{p^{+}-q^{+}} e^{i(\hat{p}-\hat{q}) \cdot x}+\frac{d^{\dagger}\left(q^{+}\right) d\left(p^{+}\right)}{p^{+}-q^{+}} e^{-i(\hat{p}-\hat{q}) \cdot x}+\right. \\
+ & \left.\frac{b^{\dagger}\left(p^{+}\right) d^{\dagger}\left(q^{+}\right)}{p^{+}+q^{+}} e^{i(\hat{p}+\hat{q}) \cdot x}+\frac{b\left(q^{+}\right) d\left(p^{+}\right)}{p^{+}+q^{+}} e^{-i(\hat{p}+\hat{q}) \cdot x}\right],
\end{aligned}
$$

and similarly for $\Sigma(x)$. The LF and SL Hamiltonians of the model have a different structure:

$$
\begin{aligned}
& P^{-}=\int_{-\infty}^{+\infty} \mathrm{d} x\left\{m\left[\Psi_{1}^{\dagger}(x) \Psi_{2}(x)+\Psi_{2}^{\dagger}(x) \Psi_{1}(x)\right]+M\left[\Phi_{1}^{\dagger}(x) \Phi_{2}(x)+\Phi_{2}^{\dagger}(x) \Phi_{1}(x)\right]\right\}, \\
& H_{\text {int }}=-g \int_{-\infty}^{+\infty} \mathrm{d} x\left(j^{0}(x) J^{1}(x)-j^{1}(x) J^{0}(x)\right) .
\end{aligned}
$$

$H_{\text {int }}$ consists of sixteen four-fermion terms of the form $F_{6}(p, q, r) b^{\dagger}(p) d^{\dagger}(q) a^{\dagger}(r) c^{\dagger}(-p-q-r)$, $F_{6}(p, q, r)=f_{2}(p, q) f_{2}(r, r-p-q)-f_{1}(p, q) f_{1}(r, r-p-q)$, e.g. Let us try to transform the Hamiltonian (5.7) to a bilinear form analogously to the Thirring model. For the operator $B$ we get, writing

$$
\begin{aligned}
& j^{0}(x)=\int_{-\infty}^{+\infty} \frac{d k}{2 \pi}\left[B(k, t) e^{i k x}+B^{\dagger}(k, t) e^{-i k x}\right], j^{1}(x)=\int_{-\infty}^{+\infty} \frac{d k}{2 \pi}\left[D(k, t) e^{i k x}+D^{\dagger}(k, t) e^{-i k x}\right] \\
& J^{0}(x)=\int_{-\infty}^{+\infty} \frac{d k}{2 \pi}\left[A(k, t) e^{i k x}+A^{\dagger}(k, t) e^{-i k x}\right], J^{1}(x)=\int_{-\infty}^{+\infty} \frac{d k}{2 \pi}\left[C(k, t) e^{i k x}+C^{\dagger}(k, t) e^{-i k x}\right]
\end{aligned}
$$




$$
\begin{aligned}
B(k, t)=\int_{-\infty}^{+\infty} & d p\left\{\theta ( k p ) \left[f_{1}(p, p+k)\left(b^{\dagger}(p) b(p+k)-d^{\dagger}(p) d(p+k)\right) e^{i(E(p)-E(p+k)) t}-\right.\right. \\
& \left.-f_{2}(-p, p+k)(b(-p) d(p+k)+d(-p) b(p+k)) e^{-i(E(p)+E(p+k)) t}\right]+ \\
& +\theta(p(k-p))\left[f_{2}(p, k-p) d(p) b(k-p) e^{-i(E(p)+E(k-p)) t}+\right. \\
& \left.\left.+\frac{1}{2} f_{1}(p, p-k)\left(b^{\dagger}(p-k) b(p)-d^{\dagger}(p-k) d(p)\right) e^{i(E(p-k)-E(p)) t}\right]\right\}
\end{aligned}
$$

and similarly for the operators $A, C, D$. The Hamiltonian becomes bilinear:

$$
\begin{gathered}
H_{\text {int }}=-\frac{g}{\pi} \int_{-\infty}^{+\infty} d k\left[B^{\dagger}(k) C(k)+C^{\dagger}(k) B(k)+B^{\dagger}(k) C^{\dagger}(-k)+B(k) C(-k)\right. \\
\left.-A^{\dagger}(k) D(k)-D^{\dagger}(k) A(k)-A^{\dagger}(k) D^{\dagger}(-k)-A(k) D(-k)\right] .
\end{gathered}
$$

For its diagonalization by a Bogoliubov transformation, one has to know the commutators like $\left[B(k), B^{\dagger}(l)\right]$, e.g. Will the correlation functions simplify when calculated with the physical vacuum obtained from the diagonalization? They were found to be surprisingly complicated [3]. Indeed, the SL operator solutions $\Psi^{I(I I)}(x)=\vdots \exp \left[\mp 2 \pi^{1 / 2} i g \sigma^{I(I I)}(x)\right] \vdots \psi^{I(I I)}(x)$ yield for example

$$
\begin{aligned}
& \left\langle: \psi_{2}^{I \dagger \dagger}(x) \psi_{1}^{I I}(x):: \exp \left[-2 \pi^{1 / 2} i g \sigma^{I I}(0)\right]:\right\rangle_{0}= \\
& =\frac{2 m \sin \pi g}{\pi^{2}} K_{g}^{2}\left(m\left(-x^{2}\right)^{1 / 2}\right)-\frac{2 m \sin \pi g}{\pi^{2}} \int_{0}^{\infty} \frac{\cosh (2 g-1) \beta}{\cosh \beta} K_{0}\left(2 m\left(-x^{2}\right)^{1 / 2} \cosh \beta\right) d \beta .
\end{aligned}
$$

To summarize, soluble massive models in two dimensions represent a good testing ground for a detailed comparison of the structure of the SL and LF field theory. To accomplish this goal, one has to find the true ground state of the SL versions of the models. The next step will be to derive commutators between the composite operators $B, B^{\dagger}, C, C^{\dagger} \ldots$, to diagonalize the Hamiltonian (5.10) and to calculate the correlation functions of the type (5.11) using the found nonperturbative vacuum. A similar treatment can be applied to the derivative-coupling model of Schroer [8].

\section{Acknowledgments}

This work was supported by the grant No. APVT 51-005704 of the Slovak Research and Development Agency, by the VEGA grant No. 2/6068/2006 and by the Slovak CERN Commission.

\section{References}

[1] G.McCartor and D. G. Robertson, Z. Phys. C53 (1992) 661.

[2] H. Lehmann and K. Stehr, preprint DESY 76/29 (1976).

[3] B. Schroer, T. T. Truong and P. Weisz, Annals of Physics 102 (1976) 156.

[4] B. Klaiber, in Boulder 1974, Lectures in Theoretical Physics Vol. Xa, New York (1968) 141.

[5] A. S. Wightman, in High Energy Interactions and Field Theory, Cargese Lect. in Theor. Phys. (1964).

[6] P. Federbush, Phys. Rev. 121 (1961) 1247.

[7] D. C. Mattis and E. H. Lieb, J. Math. Phys. 6 (1965) 304.

[8] B. Schroer, Fortsch. Physik 11 (1963) 1. 